\title{
Complexity Approaches to Complexity Measurement of the Production Process
}

\author{
Zuzana Soltysova ${ }^{1, *}$, Slavomir Bednar ${ }^{1}$, Annamaria Behunova ${ }^{1}$ \\ ${ }^{1}$ Technical University of Kosice, Faculty of Manufacturing Technologies with a seat in \\ Presov, Bayerova 1, 08001 Presov, Slovakia \\ zuzana.soltysova@tuke.sk, slavomir.bednar@tuke.sk, annamaria.behunova@tuke.sk
}

\begin{abstract}
The paper identifies and quantifies static complexity indices of the two layout types: job shop and cellular layout. Subsequently, both layout types are compared in terms of static complexity and conclusions are made towards the research question stated. As it was demonstrated on the two production layout models, lower complexity in the case of the flow shop production system has been obtained.
\end{abstract}

Keywords: structural complexity; job shop; cellular layout; manufacturing; measure

\section{Introduction}

A number of authors, so far have performed measurements of complexity within manufacturing systems with a use of various methods. Their most frequent finding was that in the case of job shop layout, complexity of the production reaches higher values than in the case of flow shop production layout [1-5]. Complexity is often considered as a negative aspect of all types of activities, as it generally negatively affects efficiency of a production system [6-8].

Each production system can be described operational, dynamic complexity [9-12]. Operational complexity is linked with measurement of dynamical aspects of a production system during its operation. Therefore, this research will adopt principles of static (structural) complexity measurement as it appears as a result of various production system's layouts.

We will consider two individual layout cases, with technologically oriented production system (job shop), and with batch production system (flow shop). The aim of this research is to assess which production system layout has higher structural complexity. With respect to this research aim, the following research (RQ) question must be answered:

$R Q$ : Will the assumption of lower complexity of the flow shop layout be confirmed also using our proposed metric?

As it was stated in the RQ, we assume the lower complexity of the flow shop, and with higher structural complexity of the job shop machine layout, as it was mentioned in the works of other authors [13-14] using various types of structural-static complexity metrics.

The research will be performed via the assessment of structural complexity of production systems using six individual complexity coefficients, applying specific 
methodologies [2]. Each of the measures refers to characteristics of each layout and its properties. These characteristic include: number of links between work-places, paths, cycles, decision-points and redundant paths. All the mentioned characteristics influence the complexity of production systems.

\section{Approaches to Assessment of Structural Complexity of Production Systems}

At this stage, we aim to replace "more complex" production system design (layout) with a "less complex" system arrangement, while nodes with pre-defined inputs and outputs, material flow and its direction, are retained. The initial node refers to the beginning of the production process and of the material flow, while the end-node is the end of both, production process and material flow. Each node within a diagram is a representation of a workstation, while each workstation has at least input and output flows (except for two - initial and end nodes).

The subsequent step was to create a matrix of relation (M). Such matrix shows important relationships between nodes based on the existence of a relation. If there is a link between nodes, the elementary matrix value is equal to " $1 "$ and if such a relationship does not exist in a matrix, then the value is equal to "0". Arrangement of the nodes in the matrix is the sequence of material flow from the start till the end node.

An important step is the calculation of complexity indicators, or of so called complexity indices.

The formulas of all complexity indices:

Density Index (D)

$$
D=\frac{k}{n(n-1)}
$$

where $k$-is the number of links; and $n$ - is the total number of nodes (representing

workstations).

Path Index (P)

$$
P=1-\frac{p}{N}
$$

where $p$ - is the maximum theoretical number of paths; $N$ - the number of all existing paths.

Cycle Index (C)

$$
C=\frac{c}{M C},
$$

where $c$ - is the number of valid cycles; and $M C$ - is the maximum theoretical number of cycles.

where $n$-in the number of nodes; $i=2$.

$$
M C=\sum_{i=2}^{n} C_{(n, i)},
$$

Decision Points Index (DS)

$$
D S=1-\frac{S P}{L P},
$$

where $S P$ - is the number of within the shortest possible path; $L P$ - is the number of nodes within the longest possible path.

Distribution Redundancy Index (RD) 


$$
R D=\frac{r}{a},
$$

where $r$ - is the number of redundancy states occurred between two adjacent nodes; $a$ is the maximum theoretical number of redundancy state occurrence among all adjacent nodes.

Magnitude Redundancy Index (RM)

$$
R M=\frac{p r}{w}=\frac{w-a}{w},
$$

where $p r$ - is the total number of redundant parallel arrows; $w$ - is the total number of assigned arrows; $a$ - is the number of adjacencies.

Assessment of structural complexity:

Obtained values of complexity indices will be applied to determine the overall structural complexity of a manufacturing system using the following three approaches:

Average value (A)

$$
A=\frac{1}{n} \sum_{i=1}^{n} a_{i}=\frac{a_{1}+a_{2}+a_{3}+\cdots+a_{n}}{n},
$$

where $n-$ is the total number of complexity indices obtained; $a_{i}$ is the value of individual complexity indices, where $i=1, \ldots n$.

Aggregated Complexity Index (CI)

$$
a=\frac{1}{2}\left[\left(C_{t} * C_{1}\right)+\sum_{i=1}^{i=t-1}\left(C_{i} * C_{i+1}\right)\right] \sin \left(\frac{360}{t}\right),
$$

where $C_{i}$ - is the value of individual indices, where $i=1,2, \ldots t ; t-$ is the total number of complexity indices obtained.

$$
A=\frac{t}{2} \sin \frac{360}{t}
$$

where $A$ - is the „total plot area“; $t-$ is the total number of complexity indices obtained.

In order to obtain aggregated complexity index $(\mathrm{CI})$, the following equation is used:

Vector Method (V)

$$
C I=\frac{a}{A}
$$

Vector size $\mathrm{V}$ can be determined using the Phytagorean theorem, as follows:

$$
V=\sqrt{\left(V_{t}\right)^{2}+\left(V_{u}\right)^{2}+\left(V_{w}\right)^{2}+\left(V_{x}\right)^{2}+\left(V_{y}\right)^{2}+\left(V_{z}\right)^{2}},
$$

where $V t-z-$ is, in this case, the value of individual complexity indicators.

The last step is generation of summary table to compare all complexity measures and their values against each other.

\section{Application of the Methods for the Job-shop System Layout}

We will now proceed according to the method presented above. For this purpose, let us have theoretical model of job shop production site with five machines in each group A-D representing four types of machines according to technology. Group A may represent lathes, group B as CNC machines, group $\mathrm{C}$ as drilling machines and group D as grinding machines. Then, it is necessary to transform the initial scheme of the production site (Fig. 1) into a simplified diagram (Fig. 2). 


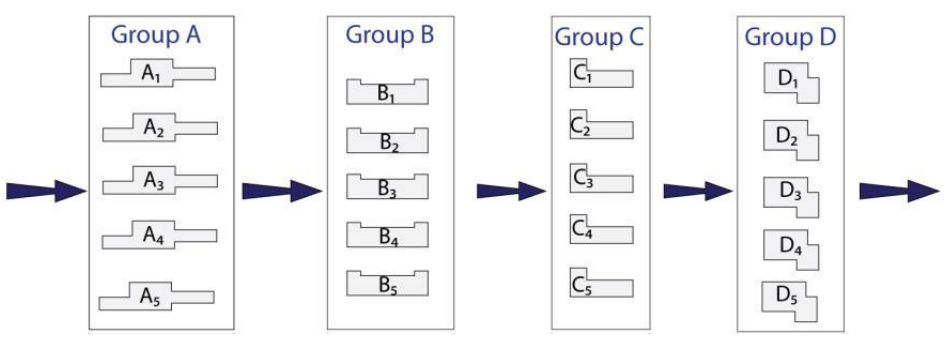

Fig. 1. Job shop arrangement of the production facility.

After obtaining a simplified diagram of the job shop production site arrangement, we get a model with 20 nodes (four groups with 5 nodes/machines), and with single input and output nodes. In this specific case, each node has five possible output paths to other technological cell (see Fig. 1). As this is technologically-based (job shop) production system, then the first five machines are very similar, or they are of the same type. These five machines have in total 25 possible output pathways to subsequent job shop cell with different type of machines. From all 25 paths, only five paths are occupied/used, while the other 20 pathways are not used, and therefore, they are redundant. The first group of machines is denoted as A, then the second by B, the third by $\mathrm{C}$ and the last group of similar machines can be denoted by $\mathrm{D}$ (see Fig. 1).

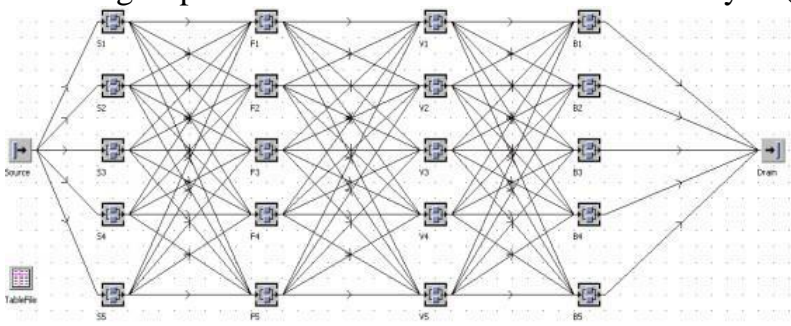

Fig. 2. A simplified diagram of the job shop production layout (generated by a simulation tool TECNOMATIX)

Generating the matrix of relations (M):

$$
\left.A M=\begin{array}{c}
\text { In } \\
A \\
A \\
B \\
C \\
C \\
D \\
\text { Out }
\end{array} \quad \begin{array}{ccccccc}
0 & 5 & 0 & 0 & 0 & 0 \\
0 & 0 & 25 & 0 & 0 & 0 \\
0 & 0 & 0 & 25 & 0 & 0 \\
0 & 0 & 0 & 0 & 25 & 0 \\
0 & 0 & 0 & 0 & 0 & 5 \\
& & 0 & 0 & 0
\end{array}\right)
$$


Calculating complexity indices:

Index D - we will take the total number of possible paths ( 75 for the whole job shop layout except for five initial and 5 output connections) and the total number of nodes ( 20 for job shop layout) into account. The number of possible paths among nodes is obtained as the sum of all arrows linking nodes $(25+25+25=75)$. Substituting the value into Eq. (1), one would obtain 0,125 .

Index $\mathbf{P}$ - considers the theoretical minimum number of paths, which is five. Each node results with five subsequent paths to group of five nodes. Each connection links single machine with five machines of subsequent group of machines, etc. The total number of possible existing paths is $15625=253$. Substituting the value into Eq. (2), one would obtain 0,9997 .

Index $\mathbf{C}$ - reflects the actual number of cycles, which is zero (cycle is the path that starts and ends in the same point and in this case, we are not talking about a cycle), and the maximum theoretical number of cycles is 33554 406. Substituting the value into Eq. (3), one would obtain 0 (zero).

Index DS -takes the total number of nodes located on the shortest possible path (from the initial till the end node, while these nodes are not counted) into account. Then, the value for the shortest possible path is four, and the value for the longest possible path is also four, so the shortest and the longest paths are equal in this production layout. Substituting the value into Eq. (5), one would obtain 0 (zero).

Index RD - gives a ratio of path occurrence between two groups of nodes with redundant paths (three paths) and the theoretical number of redundant relations among the groups of nodes (again three paths). Substituting the value into Eq. (6), the result is one.

Index RM - takes the ratio of total number of redundant arrows (oriented connections), which is 60 among the nodes, and the total number of all oriented connections among nodes, which is 75 . As there is 75 possible oriented connections among the nodes, and 25 possible connections between each group of nodes, and five of them are fixed, then the other 20 are redundant $(20+20+20=60)$. Substituting the value into Eq. (7), one would obtain 0,8 as a result.

Structural complexity and their values have been obtained by the complexity indices, using the following three approaches:

Average value (A), or the average value of complexity can be calculated from the Eq. (8), with the result of 0,488 . 
Radar chart (a) value can be obtained by substitution of complexity indices into the Eq. (9) and we get the result 1,69. To get the aggregated complexity index (CI), we must determine the relationship between a "radar area" and A "total plot area" from the Eq. (10). Then, the calculated value is 2,504. Then, the relation CI from Eq. (11) results in a value of 0,68 .

Determination of the complexity by the "vector method" by substitution into Eq. (12) returns the complexity as 1,63 .

The resulting values are shown in the following table:

Table 1. Summary of the complexity values per each index.

\begin{tabular}{lc}
\multicolumn{2}{c}{ Structural complexity indices } \\
\hline Density Index (D) & 0,125 \\
Path Index(P) & 0,9997 \\
Cycle Index(C) & 0 \\
Decision Points Index (DS) & 0 \\
Distribution Redundancy Index(RD) & 1 \\
Magnitude Redundancy Index(RM) & 0,8 \\
Average value(A) & 0,488 \\
Aggregated Complexity Index(CI) & 0,68 \\
Vector Method(V) & 1,63 \\
\hline
\end{tabular}

\section{Application of the Methods for Flow-shop System Layout}

In order to proceed towards the application of the methods to measure complexity, the original five cell-based (flow shop) production layout in Fig. 3 had to be transformed into its simplified form, as seen in Fig. 4.

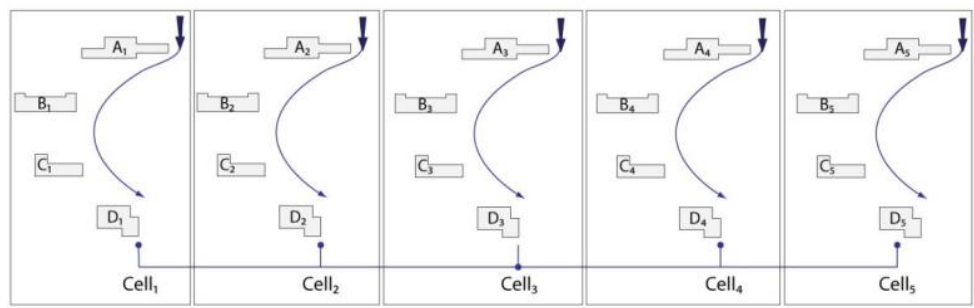

Fig. 3. Original scheme of the five cell (flow shop) production system arrangement.

Considering that the scheme in Fig. 3 above is a flow system arrangement, production machines within each of the five cells are organized based on the sequence of technological operations. The system is divided into five individual parallel cells. Each of the cells contains four different machines arranged based on the sequence of operations needed to produce the final product. Therefore, each cell contains four 
nodes, which are 25 nodes together for the whole workplace. In this special case, within each cell, there is only one path that the product can travel.

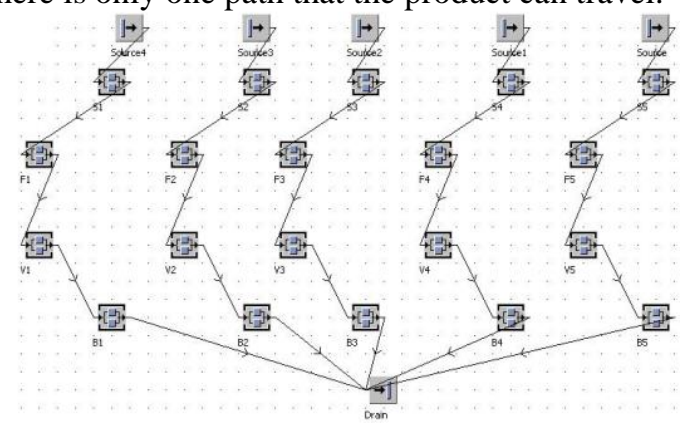

Fig. 4. A simplified diagram of the flow-shop production system using the simulation tool TECNOMATIX

To create a matrix of relations, each individual cell can be divided into four nodes (A, $\mathrm{B}, \mathrm{C}, \mathrm{D})$ with one input and one output. Then, such matrix of relations is the same for each of the five cells.

Matrix of relations (M) for the flow-shop layout:

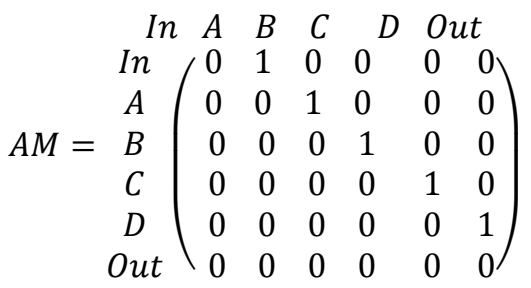

Calculating complexity indices:

Index D -takes the total number of possible paths/connections into account. In the case of the flow shop system, we have 15 possible paths out of 25 nodes. Substituting the value into Eq. (1), one would obtain 0,025.

Index $\mathbf{P}$ - takes into account the minimum theoretical number of paths (five paths) and the total number of possible existing paths is also five; substituting the value into Eq. (2), one would obtain 0 (zero).

Index $\mathbf{C}$ - considers the actual number of cycles, which equal to zero, and the maximum calculated theoretical number of cycles, which equals 33554 406; Substituting the value into Eq. (3), one would obtain 0 (zero).

Index DS - takes into account the total number of nodes located on the shortest possible path from the entry till the output node, which in this equals four, and the number of nodes located on the longest possible path, which also equals four; Substituting the value into Eq. (5), one would obtain 0 (zero). 
Index RD - takes the ratio of the number of path occurrence between any two nodes, where also redundant paths are present (zero in this case), and the total theoretical number of locations with redundant connections among nodes (equals 15 in this case); Substituting the value into Eq. (6), one would obtain 0 (zero).

Index RM - takes the ratio of the total number of redundant arrows (oriented connections), which equals zero in this case, and the total number of all connections among nodes, which equals 15; substituting the value into Eq. (7), one would obtain 0 (zero).

Again, in this case, structural complexity and their values have been obtained by the complexity indices, using the following three approaches:

Average value (A), or the average value of complexity can be calculated from the Eq. (8), with the result of 0,00417 . Radar chart (a) value can be obtained by substitution of complexity indices into the Eq. (9) and we get the result zero (0).

To get an aggregated complexity index (CI), we must again determine the relationship between a "radar area" and A "total plot area" from the Eq. (10). The calculated value is 2,504. Then, the relation CI from Eq. (11) results in a value of zero (0).

Determination of the complexity by the "vector method" by substitution into Eq. (12) returns the complexity as 0,025 .

The resulting values are shown in the following Table 2:

Table 2. Summary of the complexity values per each index of the flow shop system arrangement.

\begin{tabular}{lc}
\multicolumn{2}{c}{ Structural complexity indices } \\
\hline Density Index (D) & 0,025 \\
Path Index(P) & 0 \\
Cycle Index(C) & 0 \\
Decision Points Index (DS) & 0 \\
Distribution Redundancy Index(RD) & 0 \\
Magnitude Redundancy Index(RM) & 0 \\
Average value(A) & 0,0041 \\
Aggregated Complexity Index(CI) & 0 \\
Vector Method(V) & 0,025 \\
\hline
\end{tabular}

\section{Results and Discussion}

Mutual comparison of the structural complexity values for both, job shop and flow shop production arrangement can be seen in the following Table 3: 
Table 3. Mutual comparison of structural complexity indices.

\begin{tabular}{ccc} 
Indices & Job shop arrangement & Flow shop arrangement \\
\hline D & 0,125 & 0,025 \\
P & 0,9997 & 0 \\
C & 0 & 0 \\
DS & 0 & 0 \\
RD & 1 & 0 \\
RM & 0,8 & 0 \\
A & 0,488 & 0,00417 \\
CI & 0,68 & 0 \\
V & 1,63 & 0,025 \\
\hline
\end{tabular}

The diversity of the approach used against other approaches lies in the fact that different structural characteristics of the production system has been considered, such as: the number of paths, cycles, redundancy of mutual paths. The higher the frequency of e.g. paths, the higher the value of structural complexity.

By comparison of the results, lower complexity in the case of the flow shop production system arrangement has been demonstrated. The research question stated in the introduction of this paper can therefore be answered in the affirmative: Even using this metric, lower structural complexity of the flow shop system arrangement has been confirmed.

\section{Conclusion}

The assessment of the structural complexity is only a partial view on the complexity of the production process [15-17]. For this reason, it is not scientifically correct to state, that the general complexity of the flow production systems is lower. In order to be clear about such statement, it is needful to examine the dynamic complexity aspect of the production system, for which a methodology is not yet united.

\section{References}

1. Dima, I. C., (2010). Location and importance of logistics in the company's organisational structure. Polish Journal of Management Studies, 1, 36-43.

2. Modrak, V., Bednar, S., Modrak, J.: Static Complexity in Design of Manufacturing Systems. CIE45 Proceedings, 28-30 October 2015, Metz / France.

3. Modrak, V., Bednar, S., Marton, D.: (2015). Generating product variations in terms of mass customization. In: SAMI 2015 - IEEE 13th International Symposium on Applied Machine Intelligence and Informatics, Proceedings, 187-192 Herlany (Slovakia) 2015

4. Modrak, V., Marton, D., Bednar, S.: The impact of customized variety on configuration complexity of assembly process. Applied Mechanics and Materials 2014;474:135-40.

5. Rauch, E., Dallasega, P., \& Matt, D. T. (2016). The way from lean product development (LPD) to smart product development (SPD). Paper presented at the Procedia CIRP, 50, 2631. 
6. Espinoza Vega, V. B.: Structural Complexity of Manufacturing Systems Layout. Electronic Theses and Dissertations, 2012. Paper 5351. http://scholar.uwindsor.ca/etd/5351.

7. Production management / Marek Jančík, Anton Panda, Marcel Behún - 2012. In: Studiaimaterialy. Vol. 31, no. 1 (2012), p. 57-59. - ISSN 0860-7761 [JANČÍK, Marek PANDA, Anton - BEHÚN, Marcel]

8. Soltysova, Z., \& Bednar, S. (2015). Complexity management in terms of mass customized manufacturing. [Zarządzaniezłożonością kategoriachmasowozindywidualizowanejprodukcji] Polish Journal of Management Studies, 12(2), 139-149.

9. Frizelle, G., Suhov, Y.M.: An entropic measurement of queueing behavior in a class of manufacturing operations. Proceedings of Royal Society Series, 2001. A 457, 1579-1601.

10. Frizelle, G., Woodcock, E.: Measuring complexity as an aid to developing operational complexity. International Journal of Operations and Production Management, 1995. 15 (5), 26-39.

11. Guimaraes, T., Martensson, N., Stahre, J., Igbaria, M.: Empirically testing the impact of manufacturing system complexity on performance. International Journal of Operations \& Production Management, 1999. Vol. 19 Iss 12 pp. $1254-1269$.

12. Kovac, J., Rudy, V. (2014). Innovation production structures of small engineering production. Procedia Engineering, 96, 252-256.

13. Zhang, Z. Modeling complexity of cellular manufacturing systems, Applied Mathematical Modelling, Volume 35, Issue 9, September 2011, Pages 4189-4195, ISSN 0307-904X, http://dx.doi.org/10.1016/j.apm.2011.02.044.

14. Suzic, N., Stevanov, B., Cosic, I., Anisic, Z., Sremcev, N.: Customizing products through application of group technology: A case study of furniture manufacturing.StrojniskiVestnik/Journal of Mechanical Engineering 2012;58(12):724-31.

15. Ostertagova, E., Kovac, J., Ostertag, O., \&Malega, P. (2012). Application of morphological analysis in the design of production systems. Procedia Engineering, 48, 507-512.

16. Papakostas, N., Efthymiou, K., Mourtzis, D., \&Chryssolouris, G. (2009). Modelling the complexity of manufacturing systems using nonlinear dynamics approaches. CIRP AnnalsManufacturing Technology, 58(1), 437-440.

17. Yu, S.B., Efstathiou, J.: An Introduction of Network Complexity. In Proceedings of the Manufacturing Complexity Network Conference, 2002. Cambridge, UK. 